\title{
Computer-aided Design of a Destabilized RAFT-Adduct Radical: Towards Improved RAFT Agents for Styrene-block-Vinyl Acetate Copolymers
}

\author{
Michelle L. Coote* and David J. Henry ${ }^{\dagger}$ \\ Research School of Chemistry, Australian National University, \\ Canberra, ACT 0200, Australia
}

SUPPORTING INFORMATION

(Table S1: 7 pages total)

"To whom correspondence should be addressed, email: mcoote@rsc.anu.edu.au

${ }^{\dagger}$ Current Address: Applied Physics, School of Applied Sciences, RMIT University, GPO Box 2476V, Melbourne VIC 3001, Australia 


\section{Table S1. B3-LYP/6-31G(d) Optimized Geometries}

\section{$\mathrm{S}=\mathrm{C}(\mathrm{F}) \mathrm{SCH}_{3}$}

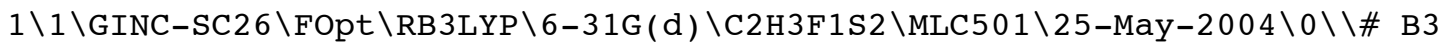
LYP/6-31G* OPT FREQ SCF=TIGHT MAXDISK=402653184 \raft_z=f_r=me-c1-6dub $3 \backslash \backslash 0,1 \backslash S, 0.6732495754,0 .,-1.5951188068 \backslash \mathrm{C}, 0.6539442516,0 ., 0.0289884936 \backslash$ $\mathrm{F}, 1.785438625,0 ., 0.7564633643 \backslash \mathrm{S},-0.6766528731,0 ., 1.1663944045 \backslash \mathrm{C},-2.118$ $3749953,0 ., 0.0535320972 \backslash \mathrm{H},-2.9969946804,0 ., 0.7038462211 \backslash \mathrm{H},-2.115457859$ $8,-0.8932702421,-0.5737748037 \backslash \mathrm{H},-2.1154578598,0.8932702421,-0.57377480$ $37 \backslash \backslash$ Version=DEC-AXP-OSF $/ 1-$ G03RevB $.03 \backslash$ State $=1-A^{\prime} \backslash \mathrm{HF}=-974.2219532 \backslash \mathrm{RMSD}=5$ $.980 \mathrm{e}-09 \backslash \mathrm{RMSF}=3.562 \mathrm{e}-05 \backslash \mathrm{Dipole}=-1.0976782,0 ., 0.3076847 \backslash \mathrm{PG}=\mathrm{CS} \quad[\mathrm{SG}(\mathrm{C} 2 \mathrm{H} 1 \mathrm{~F}$ $1 \mathrm{~S} 2), \mathrm{X}(\mathrm{H} 2) \mathrm{]} \backslash \backslash @$

\section{$\cdot \mathrm{CH}_{3}$}

$1 \backslash 1 \backslash G I N C-S C 116 \backslash F O p t \backslash U B 3 L Y P \backslash 6-31 G(d) \backslash C 1 H 3(2) \backslash R G B 501 \backslash 22-A u g-2002 \backslash 0 \backslash \backslash \# N B$ 3LYP 6-31G(D) SCF=TIGHT OPT=TIGHT FREQ MAXDISK=13107200 \methyl Opt\&Fr eq UB3-LYP $/ 6-31 \mathrm{G}(\mathrm{d}) \backslash \backslash 0,2 \backslash \mathrm{C}, 0 ., 0 ., 0 . \backslash \mathrm{H}, 1.082752351,0 ., 0 . \backslash \mathrm{H},-0.541376175$ $5,-0.937691042,0 . \backslash \mathrm{H},-0.5413761755,0.937691042,0 . \backslash \backslash$ Version=DEC-AXP-OSF / $1-\mathrm{G} 98 \mathrm{RevA} .11 .3 \backslash \mathrm{HF}=-39.8382922 \backslash \mathrm{S} 2=0.753765 \backslash \mathrm{S} 2-1=0 . \backslash \mathrm{S} 2 \mathrm{~A}=0.750007 \backslash \mathrm{RMSD}=4$. $410 \mathrm{e}-09 \backslash \mathrm{RMSF}=1.426 \mathrm{e}-09 \backslash \mathrm{Dipole}=0 ., 0 ., 0 . \backslash \mathrm{PG}=\mathrm{D} 03 \mathrm{H} \quad[\mathrm{O}(\mathrm{C} 1), 3 \mathrm{C} 2(\mathrm{H} 1)] \backslash \backslash @$

\section{$\cdot \mathrm{CH}_{2} \mathrm{OCOCH}_{3}$}

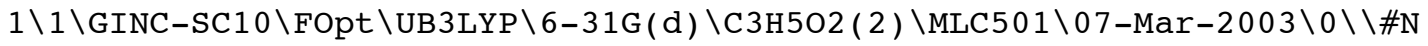

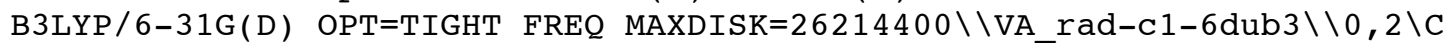
$,-1.7787811457,0.353773456,-0.0356590445 \backslash \mathrm{C},-0.2730471777,0.3716029248$, $0.0126778637 \backslash 0,0.4350619367,1.3462970692,0.0902212033 \backslash 0,0.2185047767,-$ $0.9118545746,-0.0469137652 \backslash \mathrm{H},-2.1541016217,1.3743705621,0.0441727311 \backslash \mathrm{C}$ $, 1.5760766477,-1.0845083545,-0.0455384982 \backslash \mathrm{H}, 2.202147411,-0.2279043038$, $0.1585273774 \backslash \mathrm{H}, 1.8766726719,-2.1166078394,0.0561765013 \backslash \mathrm{H},-2.1785837176$ $,-0.2564307074,0.7808932818 \backslash \mathrm{H},-2.1201583965,-0.0941758259,-0.975111322$ $\backslash \backslash$ Version=DEC-AXP-OSF $/ 1-G 98$ RevA. $11.3 \backslash \mathrm{HF}=-267.7202621 \backslash \mathrm{S} 2=0.753529 \backslash \mathrm{S} 2-1=$ $0 . \backslash \mathrm{S} 2 \mathrm{~A}=0.750008 \backslash \mathrm{RMSD}=8.716 \mathrm{e}-09 \backslash \mathrm{RMSF}=1.382 \mathrm{e}-06 \backslash \mathrm{Dipole}=-0.4442854,-0.574$ $2031,0.0426497 \backslash P G=C 01 \quad[X(\mathrm{C} 3 \mathrm{H} 5 \mathrm{O} 2)] \backslash \backslash @$

\section{$\mathrm{CH}_{3} \mathrm{SC} \cdot(\mathrm{F}) \mathrm{SCH}_{3}(1)$}

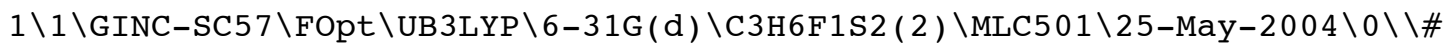

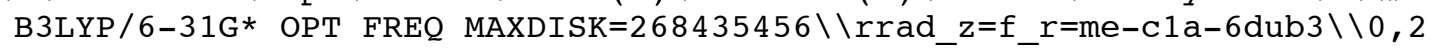
$\backslash \mathrm{H},-1.4608094972,-0.0709694822,2.7866654428 \backslash \mathrm{C},-0.3 \overline{7} 01360144,-0.0132859$ $973,2.7604246302 \backslash \mathrm{H},-0.047144627,1.0287818623,2.7724556354 \backslash \mathrm{S}, 0.28238046$ $58,-0.8622651787,1.2714810842 \backslash \mathrm{C},-0.3854096711,0.1615973635,0.012289989$ $3 \backslash S,-0.4256627666,-0.3584314418,-1.6511070005 \backslash \mathrm{C}, 1.0680954105,0.4678850$ $636,-2.3567223078 \backslash \mathrm{H}, 1.9823767235,-0.0023678665,-1.9875450804 \backslash \mathrm{H}, 1.05540$ $14148,1.5286802503,-2.1012022026 \backslash \mathrm{H}, 1.0046500744,0.3524651747,-3.441622$ $0922 \backslash \mathrm{F},-0.240367635,1.5038191257,0.2138987402 \backslash \mathrm{H}, 0.0460530895,-0.536994$ $7221,3.6242204267 \backslash \backslash$ Vers ion=DEC-AXP-OSF $/ 1-G 03 R$ RevB .03 $\backslash$ State $=2-A \backslash H F=-1014$ $.0864778 \backslash \mathrm{S} 2=0.755211 \backslash \mathrm{S} 2-1=0 . \backslash \mathrm{S} 2 \mathrm{~A}=0.750019 \backslash \mathrm{RMSD}=5.746 \mathrm{e}-09 \backslash \mathrm{RMSF}=8.505 \mathrm{e}-0$ $6 \backslash \mathrm{Dipole}=0.3608014,0.2695271,0.2839685 \backslash \mathrm{PG}=\mathrm{C} 01 \quad[\mathrm{X}(\mathrm{C} 3 \mathrm{H} 6 \mathrm{~F} 1 \mathrm{~S} 2)] \backslash \backslash @$

\section{$\mathrm{CH}_{3} \mathrm{SCH}(\mathrm{F}) \mathrm{SCH}_{3}$}

$1 \backslash 1 \backslash G I N C-S C 64 \backslash F O p t \backslash R B 3 L Y P \backslash 6-31 G(d) \backslash C 3 H 7 F 1 S 2 \backslash M L C 501 \backslash 15-F e b-2005 \backslash 0 \backslash \backslash \#$ B3 LYP/6-31G* OPT FREQ MAXDISK=268435456 MAXDISK=268435456 \\RALK_z=f_r=me $-\mathrm{C} 1 \mathrm{a}-6 \mathrm{dub} 3 \backslash \backslash 0,1 \backslash \mathrm{H},-3.0805558466,0.1303932158,-0.7908671627 \backslash \mathrm{C},-2.703844$ $4155,0.1929685141,0.2344992264 \backslash \mathrm{H},-2.5466307589,1.236087833,0.513725437$ $6 \backslash \mathrm{S},-1.1523204493,-0.7593850192,0.4322493415 \backslash \mathrm{C},-0.0737960822,0.2187978$ $253,-0.6734049093 \backslash S, 1.6407504158,-0.3857828554,-0.6724647002 \backslash \mathrm{C}, 2.12818$ 
$05575,0.0336969733,1.0425883007 \backslash \mathrm{H}, 1.6669029888,-0.637072439,1.76945892$ $27 \backslash \mathrm{H}, 1.8530713124,1.0683039758,1.2546397351 \backslash \mathrm{H}, 3.2150619299,-0.06969991$ $33,1.0857428539 \backslash \mathrm{F},-0.1295427031,1.5596663538,-0.312522299 \backslash \mathrm{H},-3.4360959$ $005,-0.2578694827,0.9089610312 \backslash \mathrm{H},-0.42398922,0.1427657436,-1.707610095$ $1 \backslash \backslash$ Version=DEC-AXP-OSF $/ 1-G 03$ RevB . 03 \State $=1-A \backslash H F=-1014.7387036 \backslash$ RMSD $=4$. $526 e-09 \backslash \mathrm{RMSF}=4.969 \mathrm{e}-06 \backslash \mathrm{Dipole}=-0.3785589,0.1901512,0.1582742 \backslash \mathrm{PG}=\mathrm{C} 01] \mathrm{X}$ ( C3H7F1S2) ] \\@

\section{$\mathrm{CH}_{3} \mathrm{SC} \cdot(\mathrm{F}) \mathrm{SCH}_{2} \mathrm{OCOCH}_{3}(4)$}

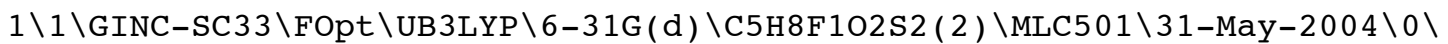
$\backslash \#$ B3LYP $/ 6-31 G *$ OPT $=($ MAXCYC=300) FREQ GEOM=CHECK GUESS=READ MAXDISK=40

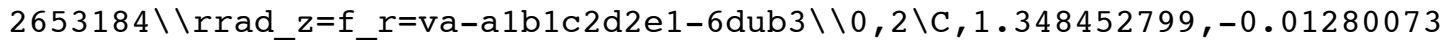
$78,0.32334631 \overline{2} 7 \backslash \mathrm{F}, 1.6683273236,-0.8665081215,1.3387796557 \backslash \mathrm{S}, 2.58689574$ $65,1.117692045,-0.1432312448 \backslash S, 0.1585089702,-0.6139234859,-0.82131261 \backslash$ $\mathrm{C}, 4.0668370788,0.0275225816,-0.3208345924 \backslash \mathrm{C},-1.1533597783,-1.160199762$ $1,0.3463735784 \backslash \mathrm{H},-1.7210213906,-1.9322685529,-0.1739449799 \backslash \mathrm{H},-0.676419$ $0801,-1.5469082514,1.2449018386 \backslash 0,-2.011595768,-0.106284409,0.77116814$ $52 \backslash C,-3.0321720436,0.2100790435,-0.0807637613 \backslash 0,-3.2320378877,-0.37315$ $94217,-1.1204010499 \backslash \mathrm{C},-3.8336916104,1.3660113838,0.4606904532 \backslash \mathrm{H}, 4.9297$ $406674,0.6909462639,-0.4181150232 \backslash \mathrm{H}, 3.9812688647,-0.6004121882,-1.2105$ $759647 \backslash \mathrm{H}, 4.1793907233,-0.5890534484,0.5723014932 \backslash \mathrm{H},-3.2166621676,2.271$ $2946324,0.4587007794 \backslash \mathrm{H},-4.7133038827,1.5215792824,-0.1645976094 \backslash \mathrm{H},-4.1$ $317445422,1.1749740023,1.4960055357 \backslash \backslash$ Version=DEC-AXP-OSF / 1-G03RevB.03\ State $=2-A \backslash H F=-1241.9601291 \backslash \mathrm{S} 2=0.755369 \backslash \mathrm{S} 2-1=0 . \backslash \mathrm{S} 2 \mathrm{~A}=0.750021 \backslash \mathrm{RMSD}=3.210$ e-09 $\backslash \mathrm{RMSF}=3.049 \mathrm{e}-06 \backslash \mathrm{Dipole}=0.292382,-0.0780562,0.5610242 \backslash \mathrm{PG}=\mathrm{C} 01] \mathrm{X}(\mathrm{C} 5 \mathrm{H}$ $8 \mathrm{~F} 102 \mathrm{~S} 2)] \backslash \backslash$ Q

\section{$\mathrm{CH}_{3} \mathrm{SCH}(\mathrm{F}) \mathrm{SCH}_{2} \mathrm{OCOCH}_{3}$}

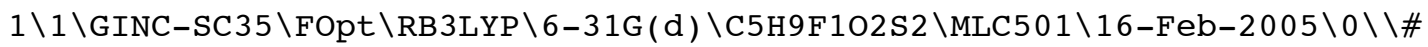
B3LYP/6-31G* OPT FREQ MAXDISK=268435456\\RALK z=f r=va-a1b1c2d2e1-6dub $3 \backslash \backslash 0,1 \backslash \mathrm{C}, 1.2716694096,0.6094173644,0.20931920 \overline{2} 5 \backslash \mathrm{F}, 1.5502692921,0.53921$ $15385,1.5689582379 \backslash \mathrm{S}, 2.795366607,0.7471075025,-0.7667132749 \backslash \mathrm{S}, 0.262353$ $726,-0.842662097,-0.2816561723 \backslash \mathrm{C}, 3.6741945067,-0.7630875872,-0.2172922$ $772 \backslash \mathrm{C},-1.0804976477,-0.6748550649,0.9478817029 \backslash \mathrm{H},-1.5806340726,-1.6426$ $990709,0.9962352734 \backslash \mathrm{H},-0.647782239,-0.3994611007,1.9082397703 \backslash 0,-2.018$ $6934602,0.3555173596,0.6271386227 \backslash \mathrm{C},-3.0523858855,-0.0066764477,-0.190$ $1787967 \backslash 0,-3.2162928,-1.1322660491,-0.5977503136 \backslash \mathrm{C},-3.926504933,1.1840$ $174082,-0.494359177 \backslash \mathrm{H}, 4.7016678499,-0.6638071988,-0.5753028958 \backslash \mathrm{H}, 3.227$ $3382242,-1.6661813856,-0.6367224719 \backslash \mathrm{H}, 3.6732093544,-0.8104268347,0.873$ $1429859 \backslash \mathrm{H},-3.3708485028,1.8997561925,-1.1102574651 \backslash \mathrm{H},-4.8151250982,0.8$ $53143655,-1.0327110376 \backslash \mathrm{H},-4.2108059353,1.6971889227,0.429264853 \backslash \mathrm{H}, 0.70$ $80688431,1.5295519661,0.0340676025 \backslash \backslash$ Version=DEC-AXP-OSF / 1-G03RevB.03 \S tate $=1-\mathrm{A} \backslash \mathrm{HF}=-1242.6138199 \backslash \mathrm{RMSD}=5.888 \mathrm{e}-09 \backslash \mathrm{RMSF}=3.264 \mathrm{e}-05 \backslash \mathrm{Dipole}=0.00871$ $46,0.4340701,0.4199935 \backslash \mathrm{PG}=\mathrm{C} 01[\mathrm{X}(\mathrm{C} 5 \mathrm{H} 9 \mathrm{~F} 102 \mathrm{~S} 2)] \backslash \backslash @$

\section{$\mathrm{S}=\mathrm{C}(\mathrm{H}) \mathrm{SCH}_{3}$}

$1 \backslash 1 \backslash G I N C-S C 28 \backslash F O p t \backslash R B 3 L Y P \backslash 6-31 G(d) \backslash C 2 H 4 S 2 \backslash M L C 501 \backslash 16-J u 1-2002 \backslash 1 \backslash \backslash \# P$ RB3 LYP /6-31G * FOPT $=($ TIGHT, MAXCYC $=150$, Z-MATRIX $)$ MAXDISK $=131072000$ FREQ SCF $=\mathrm{TIGHT} \backslash \backslash \mathrm{S}=\mathrm{C}(\mathrm{H})-\mathrm{S}-\mathrm{Me} \mathrm{CS}(2 \mathrm{a}) \backslash \backslash 0,1 \backslash \mathrm{S} \backslash \mathrm{C}, 1, \mathrm{~b} 1 \backslash \mathrm{H}, 2, \mathrm{~b} 2,1, \mathrm{a} 1 \backslash \mathrm{S}, 2, \mathrm{~b} 3,1, \mathrm{a} 2,3,18$ 0 . , $0 \backslash \mathrm{C}, 4, \mathrm{~b} 4,2, \mathrm{a} 3,1,0 ., 0 \backslash \mathrm{H}, 5, \mathrm{~b} 5,4, \mathrm{a} 4,2,180 ., 0 \backslash \mathrm{H}, 5, \mathrm{~b} 6,4, \mathrm{a} 5,2, \mathrm{dh} 1,0 \backslash \mathrm{H}, 5, \mathrm{~b}$ $6,4, a 5,2,-d h 1,0 \backslash \backslash \mathrm{b} 1=1.63746361 \backslash \mathrm{b} 2=1.09330009 \backslash \mathrm{b} 3=1.73755944 \backslash \mathrm{b} 4=1.820696$ $57 \backslash \mathrm{b} 5=1.09233155 \backslash \mathrm{b} 6=1.09203481 \backslash \mathrm{a} 1=121.28809715 \backslash \mathrm{a} 2=129.63000423 \backslash \mathrm{a} 3=103$. $10279807 \backslash \mathrm{a} 4=106.35470404 \backslash \mathrm{a} 5=110.18674431 \backslash \mathrm{dh} 1=60.3841272 \backslash \backslash$ Version=DEC $-\mathrm{A}$ $\mathrm{XP}-\mathrm{OSF} / 1-\mathrm{G} 98 \mathrm{RevA} .11 .3 \backslash \mathrm{State}=1-\mathrm{A}^{\prime} \backslash \mathrm{HF}=-874.9871381 \backslash \mathrm{RMSD}=5.554 \mathrm{e}-09 \backslash \mathrm{RMSF}=5$ $.472 \mathrm{e}-06 \backslash \mathrm{Dipole}=-0.6513287,0 ., 0.6109301 \backslash \mathrm{PG}=\mathrm{CS} \quad[\mathrm{SG}(\mathrm{C} 2 \mathrm{H} 2 \mathrm{~S} 2), \mathrm{X}(\mathrm{H} 2)] \backslash \backslash @$ 


\begin{abstract}
$\mathrm{H}-\mathrm{CH}_{3}$
$1 \backslash 1 \backslash$ GINC-RSCQC2 $\backslash F O p t \backslash R B 3 L Y P \backslash 6-31 \mathrm{G}(\mathrm{d}) \backslash \mathrm{C} 1 \mathrm{H} 4 \backslash \mathrm{MICHELLE} \backslash 01-J u n-2001 \backslash 1 \backslash \backslash \# \mathrm{P}$ R B3LYP $/ 6-31 \mathrm{G} *$ OPT=Z-MATRIX FREQ=NORAMAN MAXDISK $=262144000 \backslash \backslash$ METHANE TEST $\backslash \backslash 0,1 \backslash \mathrm{C} \backslash \mathrm{H}, 1, \mathrm{bl} \backslash \mathrm{H}, 1, \mathrm{bl}, 2,109.47122063 \backslash \mathrm{H}, 1, \mathrm{bl}, 2,109.47122063,3,120 ., 0 \backslash \mathrm{H}$, $1, \mathrm{bl}, 2,109.47122063,3,-120 ., 0 \backslash \backslash \mathrm{bl}=1.09326575 \backslash \backslash$ Version=IBM-RS6000-G98Re $\mathrm{VA} .9 \backslash \mathrm{HF}=-40.5183892 \backslash \mathrm{RMSD}=1.077 \mathrm{e}-09 \backslash \mathrm{RMSF}=1.309 \mathrm{e}-08 \backslash \mathrm{Dipole}=0, .0 ., 0 . \backslash \mathrm{PG}=\mathrm{T}$ D $[\mathrm{O}(\mathrm{C} 1), 4 \mathrm{C} 3(\mathrm{H} 1)] \backslash \backslash \mathrm{e}$
\end{abstract}

\title{
$\mathrm{F}-\mathrm{CH}_{3}$
}

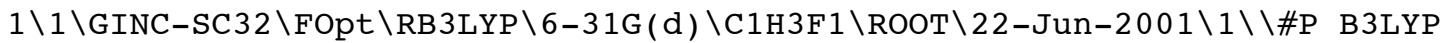
$/ 6-31 \mathrm{G}(\mathrm{D})$ OPT $=(\mathrm{Z}$-MATRIX, TIGHT $)$ FREQ MAXDISK $=3932160000 \backslash \backslash \mathrm{CH} 3 \mathrm{~F}-\mathrm{C} 3 \mathrm{~V} \backslash \backslash 0,1$ $\backslash \mathrm{C} \backslash \mathrm{F}, 1, \mathrm{Cf} \backslash \mathrm{H}, 1, \mathrm{ch}, 2, \mathrm{fCh} \backslash \mathrm{H}, 1, \mathrm{ch}, 2, \mathrm{fCh}, 3,120 ., 0 \backslash \mathrm{H}, 1, \mathrm{ch}, 2, \mathrm{fch}, 3,-120 ., 0 \backslash \backslash \mathrm{C}$ $\mathrm{f}=1.38315398 \backslash \mathrm{ch}=1.09639705 \backslash \mathrm{f} \mathrm{ch}=109.61012707 \backslash \backslash$ Version=DEC-AXP-OSF $/ 1-G 98$ RevA.9 $\backslash \mathrm{HF}=-139.733916 \backslash \mathrm{RMSD}=7.738 \mathrm{e}-09 \backslash \mathrm{RMSF}=5.739 \mathrm{e}-07 \backslash \mathrm{Dipole}=0,, 0,,-0.67$ $44796 \backslash P G=C 03 V \quad[\mathrm{C} 3(\mathrm{C} 1 \mathrm{~F} 1), 3 \mathrm{SGV}(\mathrm{H} 1)] \backslash \backslash @$

The following species [taken from Coote, M. L.; Henry, D. J. Macromolecules 2005, 38, 1415-1433] relate to RAFT agents with $\mathrm{CH}_{3}$ and $\mathrm{OCH}_{3} \mathrm{Z}$-substituents, and are

\section{included in the present work for purposes of comparison.}

\begin{abstract}
$\mathrm{S}=\mathrm{C}\left(\mathrm{OCH}_{3}\right) \mathrm{SCH}_{3}$

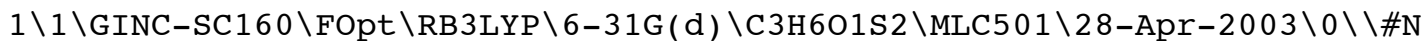
B3LYP $/ 6-31 \mathrm{G}$ (D) OPT $=($ TIGHT, MAXCYC $=100)$ FREQ MAXDISK $=131072000$ GEOM=CHEC $K$ GUESS $=$ READ $\backslash \backslash \operatorname{RAFT}(\mathrm{Z}=\mathrm{OMe} \mathrm{R}=\mathrm{CH} 3)$ lowest $\mathrm{a} 1 \mathrm{~b} 1$ in $\mathrm{Cs}$ symm $\backslash \backslash 0,1 \backslash \mathrm{C}, 0.374209$ $3441,-2.4921458868,0 . \backslash S, 1.301426637,-0.9260065359,0 . \backslash C,-0.0000019362,0$ $.2785978098,0 . \backslash S,-1.6256039629,-0.0059529119,0 . \backslash 0,0.5982011851,1.47426$ $53379,0 . \backslash \mathrm{C},-0.2142814517,2.6603482395,0 . \backslash \mathrm{H}, 1.1323627109,-3.280322659,0$ $. \backslash \mathrm{H},-0.2490760809,-2.5725262915,0.8922739391 \backslash \mathrm{H},-0.2490760809,-2.572526$ $2915,-0.8922739391 \backslash \mathrm{H}, 0.4987378961,3.485149722,0 . \backslash \mathrm{H},-0.8456382241,2.688$ $326503,-0.8916364756 \backslash \mathrm{H},-0.8456382241,2.688326503,0.8916364756 \backslash \backslash$ Version $=\mathrm{DEC}-\mathrm{AXP}-\mathrm{OSF} / 1-\mathrm{G} 98 \mathrm{RevA} .11 .3 \backslash \mathrm{State}=1-\mathrm{A}^{\prime} \backslash \mathrm{HF}=-989.5242355 \backslash \mathrm{RMSD}=5.328 \mathrm{e}-09 \backslash$ $\mathrm{RMSF}=3.625 \mathrm{e}-06 \backslash \mathrm{Dipole}=0.2790653,0.1622872,0 . \backslash \mathrm{PG}=\mathrm{CS} \quad[\mathrm{SG}(\mathrm{C} 3 \mathrm{H} 2 \mathrm{O} 1 \mathrm{~S} 2), \mathrm{X}(\mathrm{H} 4)$ ]$\backslash \backslash @$
\end{abstract}

\section{$\mathrm{CH}_{3} \mathrm{SC} \cdot\left(\mathrm{OCH}_{3}\right) \mathrm{SCH}_{3}(2)$}

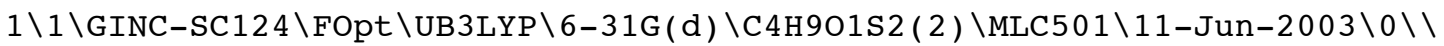
\# B3LYP $/ 6-31 \mathrm{G} *$ OPT $=($ MAXCYC $=100)$ FREQ MAXDISK $=13107200$ GEOM=CHECK GUESS $=R E A D \backslash \backslash \mathrm{rrad} z=$ ome $r=m e-a 1 b 1 c 1 d 3 e 2 f 1-6$ dub3 $\backslash \backslash 0,2 \backslash S,-1.3410354108,-1.1021$ $645587,0.00 \overline{8} 37807 \overline{2} 2 \backslash \mathrm{C},-0.0668314555,0.0846927765,-0.2625382649 \backslash \mathrm{S}, 1.525$ $0665723,-0.5327357432,-0.7113926887 \backslash \mathrm{C}, 2.3426793192,-0.7855867589,0.929$ $7015005 \backslash \mathrm{C},-2.8220656021,-0.0301869566,0.1187230656 \backslash 0,-0.1829188202,1.2$ $334385003,0.4787862469 \backslash \mathrm{C}, 0.3588901181,2.4188570239,-0.1145681207 \backslash \mathrm{H},-2$. $9779210372,0.5082852285,-0.8196451416 \backslash \mathrm{H},-3.670068575,-0.6955390127,0.2$ $998754487 \backslash \mathrm{H},-2.72432788,0.675718492,0.9443720255 \backslash \mathrm{H}, 2.2450048802,0.1147$ $964264,1.5398219577 \backslash \mathrm{H}, 3.400214885,-0.9780596806,0.7296543233 \backslash \mathrm{H}, 1.90496$ $92578,-1.6399132978,1.4510056405 \backslash \mathrm{H}, 0.1716693923,3.2223284691,0.6006696$ $643 \backslash \mathrm{H}, 1.4348670988,2.3181216146,-0.2907133132 \backslash \mathrm{H},-0.1415903241,2.638502$ $0801,-1.0650057993 \backslash \backslash$ Version=DEC-AXP-OSF $/ 1-G 03$ RevB $.03 \backslash$ State $=2-A \backslash H F=-102$ $9.3786222 \backslash \mathrm{S} 2=0.754676 \backslash \mathrm{S} 2-1=0 . \backslash \mathrm{S} 2 \mathrm{~A}=0.750016 \backslash \mathrm{RMSD}=3.068 \mathrm{e}-09 \backslash \mathrm{RMSF}=1.311 \mathrm{e}-$ $06 \backslash \mathrm{Dipole}=-0.1181936,0.8010544,0.3889717 \backslash \mathrm{PG}=\mathrm{C} 01[\mathrm{X}(\mathrm{C} 4 \mathrm{H} 901 \mathrm{~S} 2)] \backslash \backslash @$

\section{$\mathrm{CH}_{3} \mathrm{SCH}\left(\mathrm{OCH}_{3}\right) \mathrm{SCH}_{3}$}

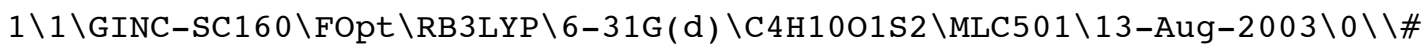
B3LYP $/ 6-31 \mathrm{G} * \mathrm{OPT}=(\mathrm{MAXCYC}=100$, TIGHT $)$ FREQ GEOM=CHECK GUESS=READ MAXDISK 
$=39321600$ INT (GRID=ULTRAFINE) \\ralk_z=ome_r=me-a1b1c1d3e2f $1-6$ dub3 $\backslash \backslash 0,1$ $\backslash S,-1.2638080102,-1.0946066938,-0.0 \overline{3} 88623 \overline{5} 7 \backslash \mathrm{C},-0.0661509511,0.22262651$ $51,-0.4773860228 \backslash \mathrm{S}, 1.6208062742,-0.506946341,-0.6859074301 \backslash \mathrm{C}, 1.9762100$ $951,-1.0009324492,1.0416521861 \backslash \mathrm{C},-2.7837706451,-0.0914568117,0.1427433$ $606 \backslash 0,-0.1350263577,1.2360458069,0.4876130868 \backslash \mathrm{C}, 0.4025768371,2.4839329$ $534,0.0588080284 \backslash \mathrm{H},-3.067093543,0.3769686088,-0.804977898 \backslash \mathrm{H},-3.5760666$ $241,-0.7816445842,0.4449633038 \backslash \mathrm{H},-2.649622786,0.6701274942,0.912327733$ $8 \backslash \mathrm{H}, 1.6788946359,-0.1976058747,1.7188468452 \backslash \mathrm{H}, 3.0563328618,-1.15320241$ $87,1.1078188609 \backslash \mathrm{H}, 1.458985461,-1.9239787154,1.3096252981 \backslash \mathrm{H}, 0.253256020$ $9,3.1821978333,0.8850644167 \backslash \mathrm{H}, 1.4725885627,2.4014309461,-0.1672236522 \backslash$ $\mathrm{H},-0.1241541813,2.8566842465,-0.8321509639 \backslash \mathrm{H},-0.3080737866,0.620483318$ $7,-1.473787359 \backslash \backslash$ Version=DEC-AXP-OSF $/ 1-G 03$ RevB . 03 $\backslash$ State $=1-A \backslash H F=-1030.02$ $74547 \backslash \mathrm{RMSD}=3.600 \mathrm{e}-09 \backslash \mathrm{RMSF}=3.094 \mathrm{e}-07 \backslash \mathrm{Dipole}=-0.2244423,0.8750638,0.1944$ $09 \backslash P G=C 01 \quad[X(C 4 H 1001 S 2)] \backslash \backslash Q$

\section{$\mathrm{CH}_{3} \mathrm{SC} \cdot\left(\mathrm{OCH}_{3}\right) \mathrm{SCH}_{2} \mathrm{OCOCH}_{3}(5)$}

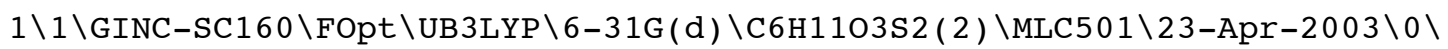
$\backslash \# \mathrm{P} \quad \mathrm{B} 3 \mathrm{LYP} / 6-31 \mathrm{G} * \mathrm{SCF}=(\mathrm{QC}, \mathrm{MAXCYC}=100) \quad \mathrm{OPT}=(\mathrm{MAXCYC}=100) \quad$ FREQ MAXDISK $=131$ $072000 \backslash \backslash \mathrm{RAFT}(\mathrm{Z}=\mathrm{OMe} \mathrm{R}=\mathrm{VA}) \mathrm{a}$ ) $1 \mathrm{c} 1 \mathrm{~d} 3 \mathrm{e} 2 \mathrm{f} 1 / / \mathrm{B} 3 \backslash \backslash 0,2 \backslash \mathrm{S}, 0.3322714222,-1.33810$ $11735,-0.9063561478 \backslash \mathrm{C}, 0.5694748971,-0.5250061298,0.6470647641 \backslash \mathrm{S}, 2.2229$ $45348,-0.384145835,1.2467459401 \backslash \mathrm{C}, 2.3646929606,-1.8402281312,2.3780173$ $258 \backslash C,-1.3096630931,-0.7061140865,-1.4131144165 \backslash 0,-0.4770788066,-0.633$ $9063631,1.515866466 \backslash \mathrm{C},-0.6921128753,0.4861332319,2.3945790952 \backslash 0,-1.229$ $3506832,0.4793060121,-2.2145907541 \backslash \mathrm{C},-1.4569891118,1.6707268399,-1.600$ $2930658 \backslash 0,-1.7749735518,1.7864051526,-0.4377061153 \backslash \mathrm{C},-1.2583630012,2.8$ $07316002,-2.5722486417 \backslash \mathrm{H},-1.7511406959,-1.470831824,-2.0528684105 \backslash \mathrm{H},-1$ $.9148450646,-0.5303100028,-0.5244913106 \backslash \mathrm{H}, 1.5016140583,-1.880452333,3$. $0454963331 \backslash \mathrm{H}, 3.2748778366,-1.6952499913,2.9659377291 \backslash \mathrm{H}, 2.4393739835,-2$ $.7685512536,1.8069091042 \backslash \mathrm{H},-1.8414714853,2.638407595,-3.4826197805 \backslash \mathrm{H},-$ $0.2036999806,2.8589667581,-2.8638987689 \backslash \mathrm{H},-1.5554863394,3.7448716146,-$ $2.1013751664 \backslash \mathrm{H},-1.5256749738,0.1957480346,3.0369865225 \backslash \mathrm{H}, 0.1974766108$, $0.681808446,3.001826327 \backslash \mathrm{H},-0.9555065988,1.3701403213,1.809273606 \backslash \backslash$ Vers ion=DEC-AXP-OSF $/ 1-G 98 R e v A .11 .3 \backslash \mathrm{HF}=-1257.2539065 \backslash \mathrm{S} 2=0.754973 \backslash \mathrm{S} 2-1=0 . \backslash \mathrm{S} 2$ $\mathrm{A}=0.750018 \backslash \mathrm{RMSD}=0.000 \mathrm{e}+00 \backslash \mathrm{RMSF}=9.849 \mathrm{e}-06 \backslash \mathrm{Dipole}=-0.3783478,0.0568044,0$ $.05398 \backslash \mathrm{PG}=\mathrm{C} 01 \quad[\mathrm{X}(\mathrm{C} 6 \mathrm{H} 1103 \mathrm{~S} 2)] \backslash \backslash \mathrm{Q}$

\section{$\mathrm{CH}_{3} \mathrm{SCH}\left(\mathrm{OCH}_{3}\right) \mathrm{SCH}_{2} \mathrm{OCOCH}_{3}$}

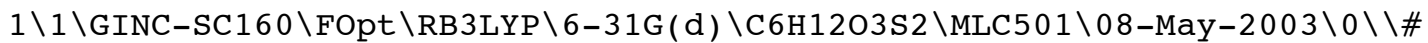
$\mathrm{B} 3 \mathrm{LYP} / 6-31 \mathrm{G} * \mathrm{OPT}=(\mathrm{MAXCYC}=100)$ FREQ MAXDISK $=134217728$ GEOM=CHECK GUESS $=$

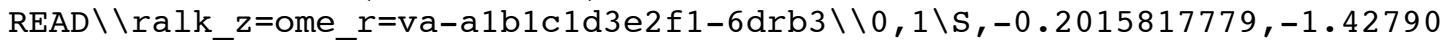
$34543,-0.1 \overline{5} 47220 \overline{2} 72 \backslash \mathrm{C},-0.9383652421,0.2179647681,0.2545016072 \backslash \mathrm{S},-2.540$ $8629392,-0.0458898946,1.1352052248 \backslash \mathrm{C},-3.5602414575,-0.7094865622,-0.23$ $33579927 \backslash \mathrm{C}, 1.238403623,-0.9376664218,-1.161139343 \backslash 0,-1.0536061247,0.96$ $03100383,-0.9216862706 \backslash \mathrm{C},-1.0525619172,2.3746501811,-0.7089736046 \backslash 0,2$. $48742376,-0.8811855308,-0.4470262731 \backslash \mathrm{C}, 2.7632003357,0.2556627682,0.232$ $7923911 \backslash 0,2.0227938086,1.2159304397,0.2779201944 \backslash \mathrm{C}, 4.1021885668,0.1537$ $864236,0.9215943715 \backslash \mathrm{H}, 1.3889552985,-1.7174692039,-1.9075110176 \backslash \mathrm{H}, 1.018$ $7806248,0.0199859847,-1.6337149931 \backslash \mathrm{H},-3.3958247807,-0.1109475676,-1.13$ $16447515 \backslash \mathrm{H},-4.6031053336,-0.6192891984,0.0803542863 \backslash \mathrm{H},-3.3287603693,-1$ $.7570678406,-0.4355418439 \backslash \mathrm{H}, 4.8779373418,-0.140195151,0.2080002027 \backslash \mathrm{H}, 4$ $.0584536804,-0.6210854801,1.6946851078 \backslash \mathrm{H}, 4.3515586415,1.1127033997,1.3$ $768834994 \backslash \mathrm{H},-1.1405520926,2.8287562082,-1.6983807588 \backslash \mathrm{H},-1.905026744,2$. $6798697453,-0.0896681835 \backslash \mathrm{H},-0.1144537416,2.6905540998,-0.2384985344 \backslash \mathrm{H}$, $-0.2974820547,0.7249720669,0.9811400417 \backslash \backslash$ Version=DEC-AXP-OSF / 1-G98RevA $.11 .3 \backslash \mathrm{HF}=-1257.9053459 \backslash \mathrm{RMSD}=2.472 \mathrm{e}-09 \backslash \mathrm{RMSF}=4.467 \mathrm{e}-06 \backslash \mathrm{Dipole}=0.5807845$, $0.2671888,-0.5051867 \backslash \mathrm{PG}=\mathrm{C} 01 \quad[\mathrm{X}(\mathrm{C} 6 \mathrm{H} 12 \mathrm{O} 3 \mathrm{~S} 2)] \backslash \backslash @$ 


\section{$\mathrm{S}=\mathrm{C}\left(\mathrm{CH}_{3}\right) \mathrm{SCH}_{3}$}

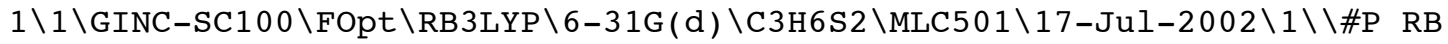
3LYP $/ 6-31 \mathrm{G} *$ FOPT $=($ MAXCYC $=150, \mathrm{Z}-$ MATRIX $)$ MAXDISK $=131072000$ FREQ SCF $=$ TIGH $\mathrm{T}$ GEOM=CHECK GUESS=READ $\backslash \backslash \mathrm{S}=\mathrm{C}(\mathrm{Me})-\mathrm{S}-\mathrm{Me} \mathrm{C} 1 \backslash \backslash 0,1 \backslash \mathrm{S} \backslash \mathrm{C}, 1, \mathrm{~b} 1 \backslash \mathrm{C}, 2, \mathrm{~b} 2,1, \mathrm{a} 1 \backslash \mathrm{S}, 2$ , b3 , 1, a2 , 3, dh $3,0 \backslash \mathrm{C}, 4, \mathrm{~b} 4,2, \mathrm{a} 3,1, \mathrm{dh} 4,0 \backslash \mathrm{H}, 5, \mathrm{~b} 5,4, \mathrm{a} 4,2, \mathrm{dh} 5,0 \backslash \mathrm{H}, 5, \mathrm{~b} 6,4, \mathrm{a} 5,2$ , dh $1,0 \backslash \mathrm{H}, 5, \mathrm{~b} 6 \mathrm{a}, 4, \mathrm{a} 5 \mathrm{a}, 2,-\mathrm{dh} 1 \mathrm{a}, 0 \backslash \mathrm{H}, 3, \mathrm{~b} 7,2, \mathrm{a} 6,1, \mathrm{dh} 6,0 \backslash \mathrm{H}, 3, \mathrm{~b} 8,2, \mathrm{a} 7,1, \mathrm{dh} 2,0$ $\backslash \mathrm{H}, 3, \mathrm{~b} 8 \mathrm{a}, 2, \mathrm{a} 7 \mathrm{a}, 1,-\mathrm{dh} 2 \mathrm{a}, 0 \backslash \backslash \mathrm{b} 1=1.64599636 \backslash \mathrm{b} 2=1.5148461 \backslash \mathrm{b} 3=1.75511783 \backslash \mathrm{b} 4=$ $1.81794969 \backslash \mathrm{b} 5=1.09307671 \backslash \mathrm{b} 6=1.0919738 \backslash \mathrm{b} 6 \mathrm{a}=1.09197036 \backslash \mathrm{b} 7=1.09324611 \backslash \mathrm{b} 8=$ $1.09537804 \backslash \mathrm{b} 8 \mathrm{a}=1.0962657 \backslash \mathrm{a} 1=122.84648556 \backslash \mathrm{a} 2=125.75342086 \backslash \mathrm{a} 3=103.776055$ $3 \backslash a 4=106.19073532 \backslash a 5=110.33849971 \backslash a 5 a=110.27255399 \backslash a 6=109.69980988 \backslash a 7=$ $112.81878244 \backslash a 7 a=109.03573022 \backslash d h 1=60.70823437 \backslash \mathrm{dh} 1 \mathrm{a}=60.13720525 \backslash \mathrm{dh} 2=167$ $.56947484 \backslash \mathrm{dh} 2 \mathrm{a}=72.03339216 \backslash \mathrm{dh} 3=181.23401229 \backslash \mathrm{dh} 4=0.90237034 \backslash \mathrm{dh} 5=180.305$ $77272 \backslash \mathrm{dh} 6=45.4338183 \backslash \backslash$ Version $=\mathrm{DEC}-\mathrm{AXP}-\mathrm{OSF} / 1-\mathrm{G} 98 \mathrm{RevA} .11 .3 \backslash \mathrm{HF}=-914.30737$ $69 \backslash \mathrm{RMSD}=3.465 \mathrm{e}-10 \backslash \mathrm{RMSF}=1.676 \mathrm{e}-06 \backslash \mathrm{Dipole}=-0.3544958,-0.0417535,0.694415$ $9 \backslash \mathrm{PG}=\mathrm{C} 01 \quad[\mathrm{X}(\mathrm{C} 3 \mathrm{H} 6 \mathrm{~S} 2)] \backslash \backslash @$

\section{$\mathrm{CH}_{3} \mathrm{SC} \cdot\left(\mathrm{CH}_{3}\right) \mathrm{S}-\mathrm{CH}_{3}(3)$}

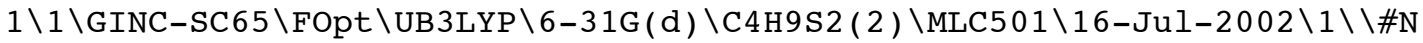
UB3LYP/6-31G(D) FOPT $=(Z-M A T R I X$, TIGHT, MAXCYC=100) FREQ MAXDISK $=13107200$ $0 \mathrm{SCF}=($ TIGHT, MAXCYC $=100)$ GEOM $=$ CHECK GUESS $=$ READ $\backslash \backslash$ mescmesme $-\mathrm{C} 1 \mathrm{~b}-6$ dub3 $\backslash \backslash 0$ , $2 \backslash \mathrm{H} \backslash \mathrm{C}, 1, \mathrm{~B} 1 \backslash \mathrm{H}, 2, \mathrm{~B} 2,1, \mathrm{~A} 1 \backslash \mathrm{S}, 2, \mathrm{~B} 3,1, \mathrm{~A} 2,3, \mathrm{D} 1,0 \backslash \mathrm{C}, 4, \mathrm{~B} 4,2, \mathrm{~A} 3,1, \mathrm{D} 2,0 \backslash \mathrm{S}, 5, \mathrm{~B} 5,4$ , A4 , 2, D3, $0 \backslash \mathrm{C}, 6, \mathrm{~B} 6,5, \mathrm{~A} 5,4,-\mathrm{D} 4,0 \backslash \mathrm{H}, 7, \mathrm{~B} 7,6, \mathrm{~A} 6,5, \mathrm{D} 5,0 \backslash \mathrm{H}, 7, \mathrm{~B} 8,6, \mathrm{~A} 7,8, \mathrm{D} 6,0 \backslash \mathrm{H}$

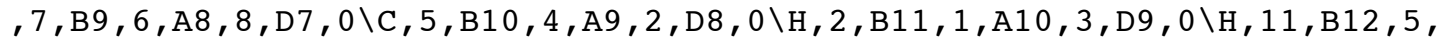
$\mathrm{A} 11,4, \mathrm{D} 10,0 \backslash \mathrm{H}, 11, \mathrm{~B} 13,5, \mathrm{~A} 12,4, \mathrm{D} 11,0 \backslash \mathrm{H}, 11, \mathrm{~B} 14,5, \mathrm{~A} 13,4, \mathrm{D} 12,0 \backslash \backslash \mathrm{B} 1=1.092657$ $93 \backslash B 2=1.09285939 \backslash B 3=1.83510395 \backslash B 4=1.77013049 \backslash B 5=1.75804982 \backslash B 6=1.832220$ $08 \backslash B 7=1.09257315 \backslash B 8=1.09085787 \backslash B 9=1.09328827 \backslash B 10=1.50447696 \backslash B 11=1.0934$ $6331 \backslash \mathrm{B} 12=1.09613505 \backslash \mathrm{B} 13=1.09553397 \backslash \mathrm{B} 14=1.10178689 \backslash \mathrm{A} 1=110.0297286 \backslash \mathrm{A} 2=11$ $0.82443615 \backslash A 3=102.23773298 \backslash A 4=118.51568336 \backslash A 5=103.96848806 \backslash A 6=110.4950$ $1693 \backslash A 7=111.21266523 \backslash A 8=105.91951214 \backslash A 9=120.43443778 \backslash A 10=109.33705389 \backslash$ $A 11=111.15729093 \backslash A 12=110.74166016 \backslash A 13=112.20742397 \backslash D 1=-124.06148497 \backslash D 2$ $=60.56470818 \backslash \mathrm{D} 3=-144.49451711 \backslash \mathrm{D} 4=-48.977946 \backslash \mathrm{D} 5=60.52936234 \backslash \mathrm{D} 6=-122.478$ $49479 \backslash D 7=118.55770463 \backslash D 8=62.40591497 \backslash D 9=119.28641302 \backslash D 10=-44.11186517 \backslash$ D1 $1=-164.00422423 \backslash D 12=75.46393503 \backslash \backslash$ Version=DEC-AXP-OSF $/ 1-G 98 R e v A .11 .3 \backslash$ $\mathrm{HF}=-954.1760596 \backslash \mathrm{S} 2=0.755169 \backslash \mathrm{S} 2-1=0 . \backslash \mathrm{S} 2 \mathrm{~A}=0.75002 \backslash \mathrm{RMSD}=5.132 \mathrm{e}-09 \backslash \mathrm{RMSF}=2$. $815 \mathrm{e}-05 \backslash \mathrm{Dipole}=0.2098476,0.5512682,-0.4657697 \backslash \mathrm{PG}=\mathrm{C} 01 \quad[\mathrm{X}(\mathrm{C} 4 \mathrm{H} 9 \mathrm{~S} 2)] \backslash \backslash @$

\section{$\mathrm{CH}_{3} \mathrm{SCH}\left(\mathrm{CH}_{3}\right) \mathrm{S}-\mathrm{CH}_{3}$}

$1 \backslash 1 \backslash$ GINC-SC50 FOpt $\backslash R B 3 L Y P \backslash 6-31 G(d) \backslash C 4 H 10 S 2 \backslash M L C 501 \backslash 16-J a n-2003 \backslash 0 \backslash \backslash \# N$ B3

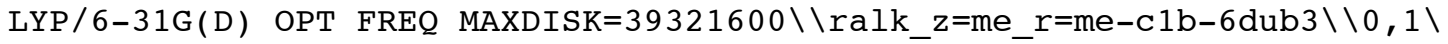
$\mathrm{H},-0.8520756361,0.8033167342,2.6879932569 \backslash \mathrm{C}, \overline{0} .237 \overline{0} 28745,0.7400719558,2$ $.5967512165 \backslash \mathrm{H}, 0.66012251,1.7461819529,2.5241712482 \backslash \mathrm{S}, 0.7265755304,-0.3$ $135602287,1.182376267 \backslash \mathrm{C},-0.0981477987,0.5907986636,-0.2087141703 \backslash \mathrm{S},-0$. $2432081378,-0.5178018206,-1.6643786617 \backslash \mathrm{C},-1.5184006495,-1.6897698027,-$ $1.08384029 \backslash \mathrm{H},-2.4735833353,-1.1832268548,-0.9147693289 \backslash \mathrm{H},-1.1948363437$ $,-2.1931652975,-0.1698746482 \backslash \mathrm{H},-1.6462270616,-2.4302742312,-1.87782798$ $13 \backslash \mathrm{C}, 0.6612927142,1.8492910624,-0.6303946624 \backslash \mathrm{H}, 0.6349852607,0.26071070$ $74,3.4953485823 \backslash \mathrm{H}, 0.7958825981,2.5207757598,0.2239981057 \backslash \mathrm{H}, 0.107849916$ $6,2.3888619535,-1.407314693 \backslash \mathrm{H}, 1.6524532115,1.5937434843,-1.0176689327 \backslash$ $\mathrm{H},-1.1090874676,0.8525173058,0.1251701442 \backslash \backslash$ Version=DEC-AXP-OSF / 1-G98Re $\mathrm{VA} .11 .3 \backslash \mathrm{HF}=-954.8250473 \backslash \mathrm{RMSD}=5.044 \mathrm{e}-09 \backslash \mathrm{RMSF}=1.205 \mathrm{e}-05 \backslash \mathrm{Dipole}=-0.682714$ $7,0.4700445,0.5466841 \backslash \mathrm{PG}=\mathrm{C} 01 \quad[\mathrm{X}(\mathrm{C} 4 \mathrm{H} 10 \mathrm{~S} 2)] \backslash \backslash @$

\section{$\mathrm{CH}_{3} \mathrm{SC} \cdot\left(\mathrm{CH}_{3}\right) \mathrm{S}-\mathrm{CH}_{2} \mathrm{COOCH}_{3}(6)$}

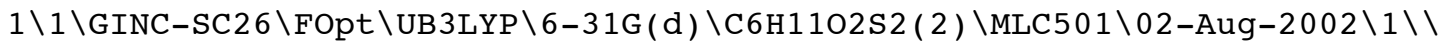
\# UB3LYP $/ 6-31 \mathrm{G} *$ OPT $=Z$-MATRIX $\mathrm{SCF}=(\mathrm{QC}, \mathrm{MAXCYC}=100, \mathrm{TIGHT})$ FREQ MAXDISK $=13$ $1072000 \backslash \backslash \mathrm{RAFT}$ RAD $(\mathrm{Z}=\mathrm{Me} \mathrm{R}=\mathrm{MA}) \mathrm{c} 1 \mathrm{p} / / \mathrm{B} 3 \backslash \backslash 0,2 \backslash \mathrm{S} \backslash \mathrm{C}, 1, \mathrm{~B} 1 \backslash \mathrm{C}, 2, \mathrm{~B} 2,1, \mathrm{~A} 1 \backslash \mathrm{S}, 2, \mathrm{~B} 3$, $1, \mathrm{~A} 2,3, \mathrm{D} 1,0 \backslash \mathrm{H}, 3, \mathrm{~B} 4,2, \mathrm{~A} 3,1, \mathrm{D} 2,0 \backslash \mathrm{H}, 3, \mathrm{~B} 5,2, \mathrm{~A} 4,5, \mathrm{D} 3, \mathrm{O} \backslash \mathrm{H}, 3, \mathrm{~B} 6,2, \mathrm{~A} 5,5, \mathrm{D} 4,0 \backslash \mathrm{C}$ 
$, 1, \mathrm{~B} 7,2, \mathrm{~A} 6,3, \mathrm{D} 5,0 \backslash \mathrm{C}, 4, \mathrm{~B} 8,2, \mathrm{~A} 7,3, \mathrm{D} 6,0 \backslash \mathrm{H}, 9, \mathrm{~B} 9,4, \mathrm{~A} 8,2, \mathrm{D} 7,0 \backslash \mathrm{H}, 9, \mathrm{~B} 10,4, \mathrm{~A} 9,2$ , D8 , 0\C , $9, \mathrm{~B} 11,4, \mathrm{~A} 10,2$, D9 , 0\0, $12, \mathrm{~B} 12,9, \mathrm{~A} 11,4, \mathrm{D} 10,0 \backslash 0,12, \mathrm{~B} 13,9, \mathrm{~A} 12,13, \mathrm{D} 1$ $1,0 \backslash \mathrm{C}, 14, \mathrm{~B} 14,12, \mathrm{~A} 13,13, \mathrm{D} 12,0 \backslash \mathrm{H}, 8, \mathrm{~B} 15,1, \mathrm{~A} 14,2, \mathrm{D} 13,0 \backslash \mathrm{H}, 8, \mathrm{~B} 16,1, \mathrm{~A} 15,16, \mathrm{D} 1$ $4,0 \backslash \mathrm{H}, 8, \mathrm{~B} 17,1, \mathrm{~A} 16,16, \mathrm{D} 15,0 \backslash \mathrm{H}, 15, \mathrm{~B} 18,14, \mathrm{~A} 17,12$, D16, 0\H , 15, B19, $14, \mathrm{~A} 18,19$

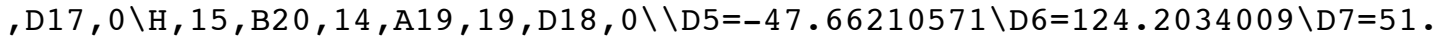
$45345692 \backslash D 8=169.97852761 \backslash D 9=-69.03688301 \backslash D 10=79.59632628 \backslash D 11=-178.3012$ $6539 \backslash D 12=-1.10736436 \backslash D 13=-176.1569452 \backslash D 14=-118.70056952 \backslash D 15=118.684277$ $86 \backslash \mathrm{D} 16=59.84254391 \backslash \mathrm{D} 17=-120.7098879 \backslash \mathrm{D} 18=119.71257159 \backslash \mathrm{B} 1=1.75470101 \backslash \mathrm{B} 2=$ $1.50144142 \backslash \mathrm{B} 3=1.7540458 \backslash \mathrm{B} 4=1.09580114 \backslash \mathrm{B} 5=1.101524 \backslash \mathrm{B} 6=1.09607085 \backslash \mathrm{B} 7=1.8$ $3909477 \backslash \mathrm{B} 8=1.85893744 \backslash \mathrm{B} 9=1.09152394 \backslash \mathrm{B} 10=1.09162639 \backslash \mathrm{B} 11=1.50894962 \backslash \mathrm{B} 12=$ $1.21538556 \backslash \mathrm{B} 13=1.34868612 \backslash \mathrm{B} 14=1.43745151 \backslash \mathrm{B} 15=1.09346144 \backslash \mathrm{B} 16=1.09284121$ $\backslash \mathrm{B} 17=1.09195971 \backslash \mathrm{B} 18=1.0931971 \backslash \mathrm{B} 19=1.09330407 \backslash \mathrm{B} 20=1.09019523 \backslash \mathrm{A} 1=122.104$ $41177 \backslash A 2=118.7219121 \backslash \mathrm{A} 3=111.19302428 \backslash \mathrm{A} 4=111.34102025 \backslash \mathrm{A} 5=111.01263129 \backslash \mathrm{A}$ $6=102.5326864 \backslash A 7=103.45710004 \backslash A 8=108.16559651 \backslash A 9=105.69370446 \backslash A 10=110$. $77782324 \backslash \mathrm{A} 11=124.84467967 \backslash \mathrm{A} 12=111.49500109 \backslash \mathrm{A} 13=115.39415873 \backslash \mathrm{A} 14=106.25$ $208603 \backslash A 15=111.51685978 \backslash A 16=109.15493098 \backslash A 17=110.56758888 \backslash A 18=110.4339$ $1008 \backslash A 19=105.51868249 \backslash D 1=179.45105199 \backslash D 2=-31.67767788 \backslash D 3=120.39887003 \backslash$ $\mathrm{D} 4=-120.58711712 \backslash \backslash$ Version=DEC-AXP-OSF / 1-G98RevA. $11.3 \backslash \mathrm{HF}=-1182.0481894 \backslash$ $\mathrm{S} 2=0.756239 \backslash \mathrm{S} 2-1=0 . \backslash \mathrm{S} 2 \mathrm{~A}=0.750029 \backslash \mathrm{RMSD}=0.000 \mathrm{e}+00 \backslash \mathrm{RMSF}=9.546 \mathrm{e}-05 \backslash \mathrm{Dipole}=$ $0.1388335,0.8127896,0.3170917 \backslash \mathrm{PG}=\mathrm{C} 01 \quad[\mathrm{X}(\mathrm{C} 6 \mathrm{H} 11 \mathrm{O} 2 \mathrm{~S} 2)] \backslash \backslash @$

\section{$\mathrm{CH}_{3} \mathrm{SCH}\left(\mathrm{CH}_{3}\right) \mathrm{S}-\mathrm{CH}_{2} \mathrm{COOCH}_{3}$}

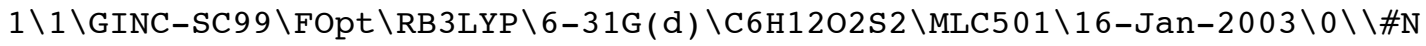

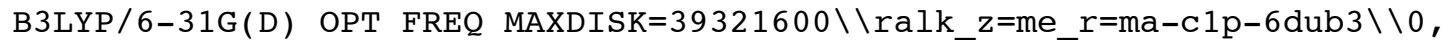
$1 \backslash \mathrm{S},-2.0997683627,0.9755983623,-0.883102629 \backslash \mathrm{C},-0.26 \overline{2} 9409913,1.20626538$ $86,-0.9107020321 \backslash \mathrm{C}, 0.1627309408,2.385076654,-1.7882723541 \backslash \mathrm{S}, 0.62129505$ $91,-0.2933816099,-1.5306066769 \backslash \mathrm{H},-0.147698331,2.2372725776,-2.82745387$ $27 \backslash \mathrm{H},-0.3051313626,3.3052065946,-1.4249666316 \backslash \mathrm{H}, 1.2512092811,2.5137187$ $413,-1.7639891476 \backslash \mathrm{C},-2.4644441444,1.5467482991,0.8200002467 \backslash \mathrm{C}, 0.122693$ $1473,-1.5416017102,-0.2782295673 \backslash \mathrm{H},-0.9664962373,-1.5163531756,-0.1828$ $477314 \backslash \mathrm{H}, 0.4461799048,-2.5081803672,-0.6689336645 \backslash \mathrm{C}, 0.7569005321,-1.25$ $72168395,1.0641991048 \backslash 0,0.3562242967,-0.4238190927,1.8552508967 \backslash 0,1.84$ $61962671,-2.0194446637,1.2741391292 \backslash \mathrm{C}, 2.5507188327,-1.768290117,2.5027$ $785393 \backslash \mathrm{H},-3.5269511465,1.3548229499,0.9930824563 \backslash \mathrm{H},-2.278745775,2.6185$ $466328,0.9342036865 \backslash \mathrm{H},-1.8703921485,0.9804471434,1.5415415076 \backslash \mathrm{H}, 1.8953$ $537217,-1.9345287758,3.3615891611 \backslash \mathrm{H}, 2.9176972252,-0.7388766263,2.52771$ $90424 \backslash \mathrm{H}, 3.3824637285,-2.4728265453,2.5105213379 \backslash \mathrm{H}, 0.0447695832,1.36550$ $2814,0.125118918 \backslash \backslash$ Version=DEC-AXP-OSF / 1-G98RevA. $11.3 \backslash \mathrm{HF}=-1182.6976795 \backslash$ $\mathrm{RMSD}=3.791 \mathrm{e}-09 \backslash \mathrm{RMSF}=5.060 \mathrm{e}-06 \backslash \mathrm{Dipole}=0.4780362,-0.0867756,0.7893997 \backslash \mathrm{PG}$ $=\mathrm{C} 01[\mathrm{X}(\mathrm{C} 6 \mathrm{H} 12 \mathrm{O} 2 \mathrm{~S} 2)] \backslash \backslash @$ 